\title{
Charged Particle Distributions in Deep Inelastic Scattering and Photoproduction
}

\section{Daniel Traynor ${ }^{* \dagger}$}

Queen Mary, University of London E-mail: d. traynore qmul . ac . uk

The study of particle production provides valuable insights into parton fragmentation and hadronisation processes and thereby helps understanding QCD in its perturbative and non-perturbative regime. In this talk fundamental tests of QCD factorisation and quark fragmentation universality are presented. Comparisons of fragmentation function results from ep scattering at HERA, both from deep inelastic scattering and photoproduction, with those from $e^{+} e^{-}$and $p \bar{p}$ experiments are shown. High precision tests of perturbative QCD predictions and of alternative resummation approaches, such as the modified leading-logarithmic approximation, are also presented. In addition a search for parton dynamics beyond DGLAP at the lowest accessible Bjorken $x$ domain at HERA is presented.

35th International Conference of High Energy Physics - ICHEP2010,

July 22-28, 2010

Paris France

\footnotetext{
*Speaker.

†n behalf of the H1 and ZEUS Collaborations.
} 


\section{Scaled momentum spectra in deep inelastic scattering at HERA}

In [1] charged particle production is studied in neutral current deep inelastic ep scattering (DIS) with the ZEUS detector at HERA using an integrated luminosity of $0.44 \mathrm{fb}^{-1}$ and in the kinematic region $10<Q^{2}<41,000 \mathrm{GeV}^{2}$. Distributions of scaled momenta in the Breit frame are presented for particles in the current fragmentation region. The evolution of these spectra with the photon virtuality, $Q^{2}$, exhibit large scaling violations. Comparing the data to $e^{+} e^{-}$results, Figure 2 left, generally supports the concept of quark-fragmentation universality. Neither Next-to-leading-order QCD coupled with fragmentation functions parameterised from $e^{+} e^{-}$data $(\mathrm{NLO}+\mathrm{FF})$ nor the modified leading-log approximation using local parton hadron duality (MLLA+LPHD) QCD calculations describe the data well. A better, albeit not prefect description is provided by the Ariadne Monte Carlo program implementing the Colour Dipole Model (CDM) of parton showers.

In addition the limiting-fragmentation hypothesis and the statistical model of Bialas and Jezabek are tested by studying the density of charged particles as a function of the pseudorapidity, $\eta_{\text {Breit }}$, over the range of $10<Q^{2}<10,240 \mathrm{GeV}^{2}$. A region of linear rise and the onset of a plateau are observed over the whole range in $Q^{2}$ and support the limiting fragmentation hypothesis. The independence of the slopes on $W$ supports the statistical approach of Bialas and Jezabek.

\section{Observation of the Hadronic Final State Charge Asymmetry in High $Q^{2}$ Deep-Inelastic Scattering at HERA}

The first measurement of the charge asymmetry of the hadronic final state at HERA in high $Q^{2}, 100<Q^{2}<8,000$, DIS is presented in [2] by the H1 collaboration. The difference between the event normalised distributions of the scaled momentum, $x_{p}$, for positively and negatively charged particles, measured in the current region of the Breit frame are shown in Figure 2 right. This charge asymmetry is found to be dependent on the scaled momentum $x_{p}$ with a larger asymmetry for large $x_{p}$. The observed charge asymmetry at large $x_{p}$ is also found to increase with the scale $Q$ corresponding at HERA to an enhancement at large Bjorken $x$. The results are consistent with the expectation that at high $x_{p}$ the asymmetry is directly related to the valence quark content of the proton. The observed charge asymmetry is reproduced by Monte Carlo models.

\section{Scaled momentum distributions of charged particles in dijet photoproduction at HERA}

In [3] the multiplicity distributions of charged particles within cones centred on jets are measured as a function of $\xi=\ln \left(1 / x_{p}\right)$, where $x_{p}$ is the fraction of the jet's momentum carried by the charged particle. These $\xi$ distributions are measured in five bins of $E_{j e t}$ and with three different cone opening angles, $\theta_{c}$, for $\gamma p$ events containing two and only two jets, using $359 \mathrm{pb}^{-1}$ of $e p$ data with the ZEUS detector.

The peak positions of the $\xi$ distributions, $\xi_{\text {peak }}$, are extracted and observed to increase roughly linearly with $\ln \left(E_{\text {jet }} \sin \left(\theta_{c}\right)\right)$ (Figure 1 left). A single value of intrinsic MLLA scale, $\Lambda_{e f f}$, is extracted by fitting the $\xi_{\text {peak }}$ data according to the predicted relationship between $\xi_{\text {peak }}$ and $\ln \left(E_{j e t} \sin \left(\theta_{c}\right) / \Lambda_{\text {eff }}\right)$. The global fit value is found to be $\Lambda_{\text {eff }}=246 \pm 3$ (stat. $)_{-8}^{+4}$ (syst. $) \mathrm{MeV}$. 

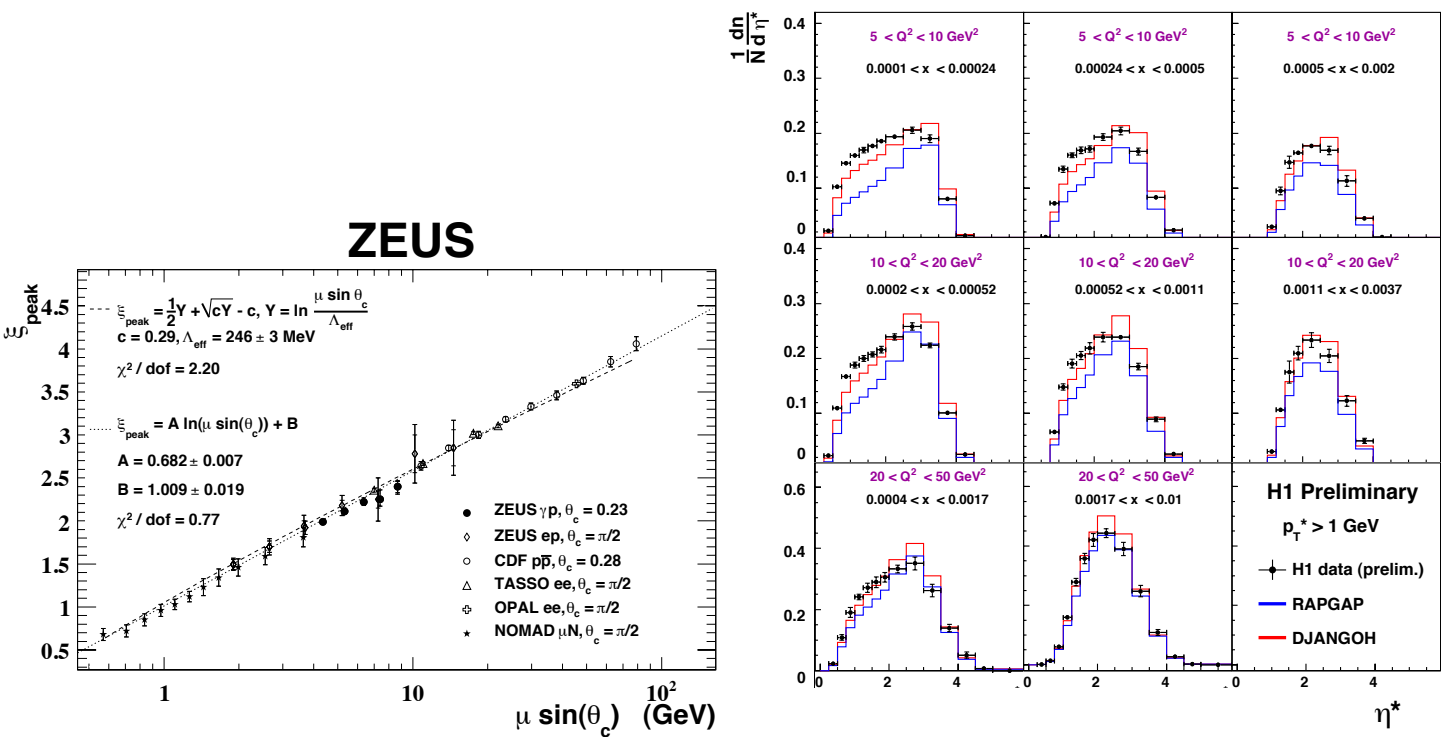

Figure 1: Left: The $\xi$ peak as a function of $\mu \sin \left(\theta_{c}\right), \mu$ is the characteristic energy scale. The ZEUS $\gamma p$ data (solid circles) and $e p$ data (diamonds) and results from OPAL (crosses), TASSO (triangles), NOMAD (stars) and CDF (open circles) collaborations are shown. Right: Measured $\eta^{*}$ for charged particles for the hadronic centre of mass system for $p_{t}^{*}>1 \mathrm{GeV}$. Data are compared to RAPGAP and DJANGOH Monte Carlo predictions.

The $E_{j e t}$ and $\theta_{c}$ dependences of $\Lambda_{e f f}$ are studied by calculating a value of $\Lambda_{e f f}$ from each $\xi_{\text {peak }}$ data point. The value of $\Lambda_{e f f}$ weakly depends on $\theta_{c}$ but no $E_{j e t}$ dependence is observed. The $\Lambda_{e f f}$ data are consistent with previously published data sets using different initial states, supporting the prediction that $\Lambda_{\text {eff }}$ is universal.

The $\xi$ distributions are also fitted using the limited momentum spectra predicted by the MLLA and assuming LPHD, in the regions where they are applicable. The theory largely described the data in these regions. The fitted MLLA functions are used to extract the value of $\Lambda_{\text {eff }}$ as a function of $E_{j e t}$ and $\theta_{c}$. The value extracted using this method with $\theta_{c}=0.23$ and averaged over $E_{j e t}$, is $\Lambda_{\text {eff }}=304 \pm 6$ (stat.) ${ }_{-32}^{+8}$ (syst.) $\mathrm{MeV}$.

The value of the LPHD parameter $\kappa_{c h}$ is extracted as a function of $E_{j e t}$ and $\theta_{c}$ from the fitted limited momentum spectra. Corrections based on next-to-MLLA theory are included. The value extracted with $\theta_{c}=0.23$ and averaged over $E_{j e t}$, is $\kappa_{c h}=0.55 \pm 0.01$ (stat.) ${ }_{-0.02}^{+0.03}$ (syst. $)_{-0.09}^{+0.11}$ (theo.). The value of $\kappa_{c h}$ has a weak dependence on $\theta_{c}$ and is consistent with the results published by the CDF collaboration. The data support the assumption that $\kappa$ is universal.

\section{Transverse momentum of charged particles at low $Q^{2}$ at HERA}

The HERA collider allows for the study of deep inelastic scattering (DIS) at very small Bjorken $x\left(10^{-5}\right)$. At such a small $x$ parton dynamics beyond DGLAP are expected to become important. In [4] the hadronic final state in deep-inelastic scattering is studied by measuring, in the hadronic centre of mass frame, the transverse momentum, $p_{t}^{*}$, and rapidity spectra, $\eta^{*}$, of charged particles in DIS with the H1 detector at HERA. The data was taken during the 2006 running period and 
corresponds to an integrated luminosity of $88.64 \mathrm{pb}^{-1}$. The analysis is performed in the kinematical region $5<Q^{2}<100 \mathrm{GeV}^{2}$ and $0.05<y<0.7$.

Figure 1 right shows the charged particle spectra as a function of $\eta^{*}$ for $p_{t}^{*}>1 \mathrm{GeV}$ in different $x$ and $Q^{2}$ regions. The measurements are compared to two QCD models corresponding to the different scenarios of the parton dynamics: the DGLAP and BFKL-like (Colour Dipole Model) evolution schemes. At large $x(x>0.001)$ both models give a satisfactory description of the data. At small $x$ the QCD model predictions by RAPGAP, based on the conventional DGLAP equations, falls below the data. Data are well described over the full kinematic ranges by the approach based on the Colour Dipole Model (DJANGOH), in which parton radiation is not ordered in $p_{T}$.

ZEUS

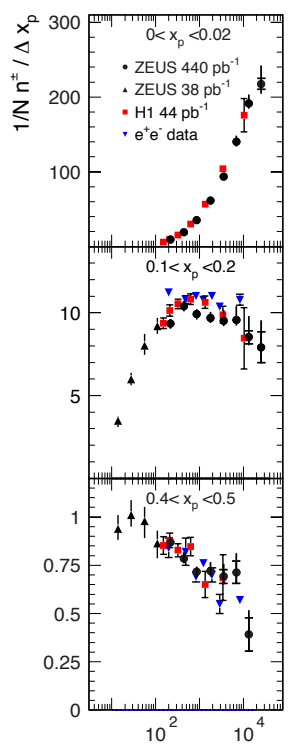

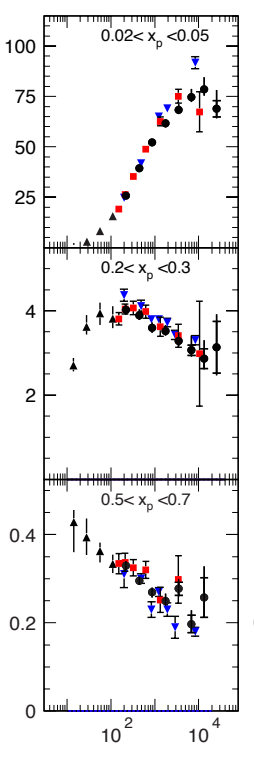
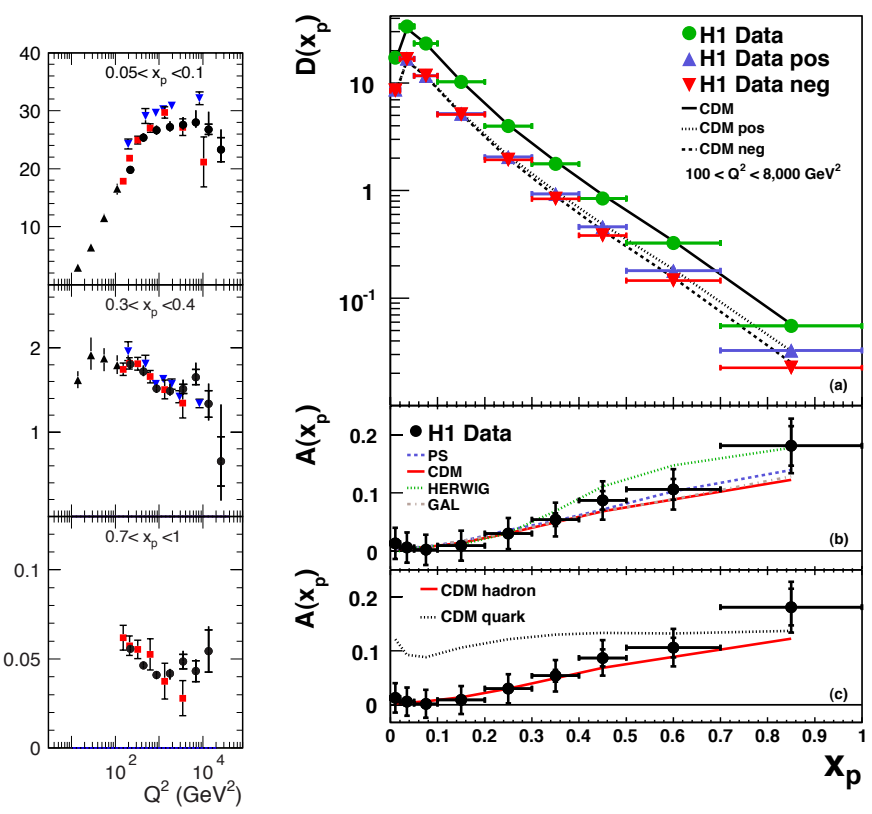

Figure 2: Left: The measured normalised distributions of the scaled momentum, $1 / N n \pm / \Delta x_{p}$, as a function of $Q$ and $x_{p}$. The dots (triangles) represent the new (previous) ZEUS measurement, the squares the H1 data and the inverted triangles the $e^{+} e^{-}$data. Right: (a) The measured normalised distributions of the scaled momentum, $D\left(x_{p}\right)$, for all charged particles and for positively (pos) and negatively (neg) charged particles separately, and (b), (c) the charge asymmetry, as a function of $x_{p}$, compared to Monte Carlo predictions.

\section{References}

[1] H. Abramowicz et al., JHEP 1006 (2010) 009 [Erratum-ibid. 1010 (2010) 030] [arXiv:1001.4026 [hep-ex]].

[2] F. D. Aaron et al. [H1 Collaboration], Phys. Lett. B 681 (2009) 125 [arXiv:0907.2666 [hep-ex]].

[3] S. Chekanov et al. [ZEUS Collaboration], JHEP 0908, 077 (2009) [arXiv:0904.3466 [hep-ex]].

[4] Anastasia Grebenyuk, talk give at the 18th International Workshop on Deep Inelastic Scattering, Florence, (2010). [H1 Collaboration] H1prelim-10-035,

http://www-h1.desy.de/h1/www/publications/htmlsplit/H1prelim-10-035.long.html. 